\title{
Atomic self-reconstruction of catalyst dominated growth mechanism of graphite structures
}

Yang $\mathrm{Hu}^{[\mathrm{a}] \dagger}$, Liu Zhu ${ }^{[\mathrm{a}] \dagger}$, Yong Peng ${ }^{[\mathrm{a}]^{*}}$, Jiecai Fu${ }^{[\mathrm{a}]}$, Xia Deng ${ }^{[\mathrm{b}]}$, Junwei Zhang ${ }^{[\mathrm{a}]}$, Hong Zhang $^{[\mathrm{a}]}$, Chaoshuai Guan ${ }^{[\mathrm{a}]}$, Abdul Karim ${ }^{[\mathrm{a}, \mathrm{d}]}$ and Xixiang Zhang ${ }^{[\mathrm{c}]}$

[a] Key Laboratory of Magnetism and Magnetic Materials of the Ministry of Education,

School of Physical Science and Technology and Electron Microscopy Centre of Lanzhou University, Lanzhou University, Lanzhou 730000, P. R. China.

[b] School of Life Science and Electron Microscopy Centre of Lanzhou University, Lanzhou University, Lanzhou 730000, P. R. China.

[c] Division of Physical Science and Engineering, King Abdullah University of Science and Technology (KAUST), Thuwal 239955, Kingdom of Saudi Arabia.

[d] Department of Physics Karakorum International University Gilgit-Baltistan, Gilgit 15100, Pakistan.

* Corresponding authors

Key Laboratory of Magnetism and Magnetic Materials of the Ministry of Education, Lanzhou University, Lanzhou 730000, P. R. China

E-mail: pengy@1zu.edu.cn (Yong Peng)

$\uparrow$ These authors contributed equally to this work. 
Abstract: Understanding the nucleation and growth process of carbon nanotubes (CNTs) is important for guiding their efficient and controllable synthesis in industry. However, the intrinsic mechanism that controls the formation of carbon nanotubes is still controversial. Here, using in-situ transmission electron microscopy (TEM), we demonstrate the dynamic catalytic growth of multilayered graphite crystallites and single-walled carbon nanotubes (SWCNTs) from the $\mathrm{Co}_{2} \mathrm{C}$ catalyst nanoparticles (NPs) at the atomic resolution. The dissociative carbon atoms arrive at the nucleation sites on the surface of small and large NPs by the surface and bulk diffusion, respectively. These two different diffusion modes are found to be the essential prerequisite for growing single-walled carbon nanotubes (SWCNTs) or multilayered graphite crystallites. The small NPs utilize crystal self-rotation to expose the (111) plane for efficiently capturing carbon atoms, while the large NPs use self-reshaping on (111) facets to provide atomic steps as active nucleation sites. Density functional theory (DFT) calculations indicate that the observations are in good agreement with the growth mechanism of graphite structures involving the preferential selectivity of crystal facets. Our results may open up the possibility of adjusting the size and crystal orientation of cobalt-based catalyst particles to efficiently synthesize the SWCNTs with high quality. 


\section{Introduction}

The growth of carbon nanotubes with controllable structure and predefined functionality has a widespread application in the field of nanotechnology. ${ }^{[1-6]}$ For the past decades, catalytic chemical vapor deposition (CVD) has been the most favored methods of CNTs' manufacture, not only in consideration of economic cost, but also the remarkable controllability in length ${ }^{[7]}$, diameter ${ }^{[8]}$, purity ${ }^{[9]}$, and transport properties ${ }^{[10,11]}$. By manipulating the composition, and pretreatment of catalysts, CNTs with narrow chirality distributions have been successfully achieved. ${ }^{[12-18]}$ These results indicate that the composition, structure and chemical environment of catalyst have a great influence on the nature of carbon nanotubes. And the interaction between the catalyst NPs and carbon source is extremely significant for the nucleation and growth of CNTs. However, in absence of an in-depth understanding about intrinsic reaction mechanisms, the precise modulation of the aspect ratio and properties of carbon nanotubes seems to be complicated. Thus, a comprehensive investigation on the growth mechanism at atomic level is highly desired.

Traditional analytical methods, including Scanning Electron Microscopy (SEM), Raman Spectrometer and selected area electron diffraction (SAED) in TEM, are employed to characterize the growth mechanism of carbon nanotubes. For example, the grown structures of carbon nanotubes are analyzed by means of characteristic peaks in Raman spectra or SAED patterns, and their growth evolution are speculated through a combination of the chemical and structural characterizations of catalyst particles. Chiang et al. ${ }^{[12]}$ found the correlation between the alloy composition and SWCNTs 
chiral distribution, deducing that the ratio of iron to nickel is a key factor to affect the chirality of SWCNTs. Yang et al. ${ }^{[17]}$ have successfully synthesized the single chirality of SWCNTs by using tungsten-based bimetallic alloy nanocrystals as catalysts, suggesting that the structural match at the interface of SWCNTs and catalyst NPs dominates their growth mechanism. Zhang et al. ${ }^{[18]}$ recently use the $\mathrm{Mo}_{2} \mathrm{C}$ and $\mathrm{WC}$ nanoparticles with uniform size as catalyst to grow the horizontal SWCNT arrays with predicted chirality, proposing that the symmetries of catalyst surface play a decisive role in determining the chirality. It is seen that the growth mechanism of SWCNTs is still in debate. Direct observation of evolution during the catalytic process at the atomistic scale and high time resolution is essential and significant for the comprehensive understanding of their intrinsic mechanism. Current in-situ TEM method can provide this capability. ${ }^{[19,20,27]}$ Environmental TEM is recently hired to discover the growth of CNTs catalyzed by Fe-group metals, which ideally provides an insight into the CNTs nucleation process and the structural evolution of catalyst NPs. ${ }^{[21-}$ ${ }^{28]}$ These experiments were typically performed at high accelerating voltages, such as 200 or $300 \mathrm{kV}$. However, it is inevitable to introduce a perturbation of high-energy electron beam radiation although the high voltage can increase the signal-to-noise ratio of images. The radiation perturbation could affect or even change the growth mechanism of the CNTs, for examples, energy barriers, the reaction threshold temperature and growth rate, which hinder us to correctly understand the intrinsic mechanisms.

In this work, we contribute an atomic observation of dynamical nucleation and 
growth processes of graphite structures catalyzed by cobalt carbide NPs by using insitu Cs-TEM heating method at low accelerating voltage. The cobalt contained carbon nanofibers (CNFs) with 250 350 $\mathrm{nm}$ diameter prepared by electrospinning technique are chosen to be the reactants, which makes the catalyst NPs to directly contact to carbon source and prevents the reduction of catalytic efficiency caused by the surface contamination. The in-situ TEM experiments were performed under $80 \mathrm{kV}$ acceleration voltage and videoed by Gatan K2 IS high speed camera to achieve a high spatial and time resolution. The electronic states of the catalyst were characterized by atomiccolumn resolution EELS to disclose the role of cobalt atoms in promoting the activation of carbon atoms. First principle calculations were further carried out to prove the preferred crystal facet of the graphite growth.

\section{Results and Discussion}

\section{The Effects of Electron Irradiation and the Experimental Design}

Electron beam and elevated temperature are considered to be the critical factors in in-situ TEM experiments. ${ }^{[29,30]}$ Compared to practical CVD experiments, there are no electrons acting on the reactants, therefore, the identification of electron beam's role is particularly important. A series of comparative experiments were performed for the comprehensive understanding of electron irradiation's effects on the growth of graphite structures. Convergent electron beam with $300 \mathrm{kV}$ accelerating voltage, which is much higher than the displacement threshold of carbon atoms $(\sim 86 \mathrm{kV})^{[31]}$, was applied onto a $\mathrm{CNF}$ at $650{ }^{\circ} \mathrm{C}$. The $\mathrm{CNF}$ was clearly observed to shrink with the diameter reducing 
from $350 \mathrm{~nm}$ to $310 \mathrm{~nm}$ within few seconds (Figure S1a, Supporting Information). Meanwhile, the multilayered graphite crystallite grew along the surface of catalyst nanoparticle (Figure S1b, Supporting Information). Conversely, when the high voltage was decreased to $80 \mathrm{kV}$ and the temperature was similarly raised up to $650{ }^{\circ} \mathrm{C}$, there is no sense of reduction in diameter of the CNF after the same irradiation time (Figure S1c, Supporting Information). And none of the multilayered graphite crystallite could be observed at the surface of catalyst NP (Figure S1d, Supporting Information). These results indicate that the effect of electron irradiation mainly lowers the threshold temperature $\left(780{ }^{\circ} \mathrm{C}\right)$ of catalytic graphitization reaction and accelerates the growth process. However, when the electron beam with $300 \mathrm{kV}$ high voltage irradiates the graphite structure at room temperature, the ultrahigh energy of the electrons results in irradiation damage, as demonstrated by the exfoliation process of graphite crystallite (Figure S2, Supporting Information).

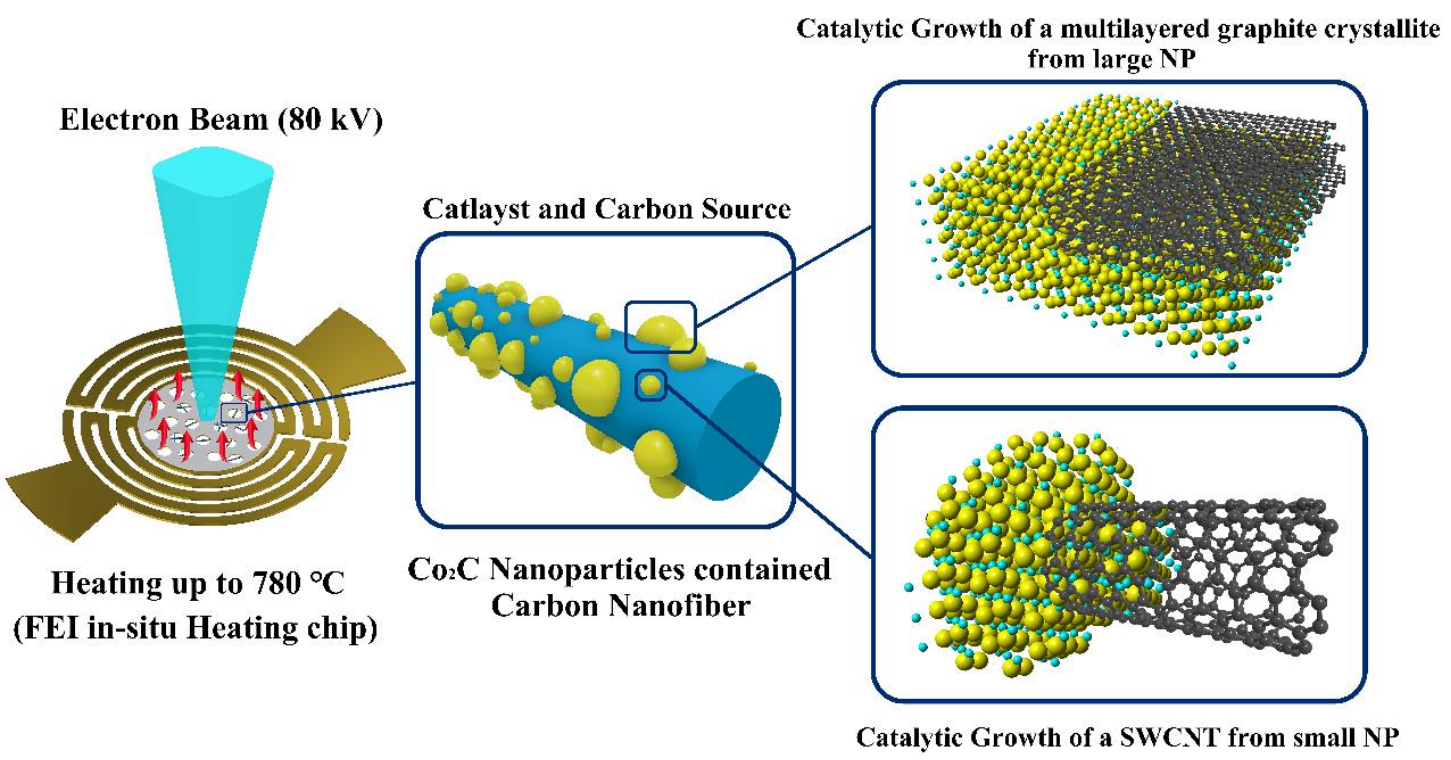

Figure 1. Conditions of in-situ heating experiment and catalytic growth of a multilayered graphite crystalline and SWCNT from $\mathrm{Co}_{2} \mathrm{C}$ catalyst nanoparticles with 
different size.

Thus, in order to avoid the irradiation damage and decouple the influence of electron beam on growth process from particle size and other parameters, $80 \mathrm{kV}$ accelerating voltage was selected to perform the in-situ Cs-TEM experiment in this work, which hopefully provides an experimental condition as close as possible to the actual CVD reaction environment. The basic idea underpinning our study is sketched in Fig. 1: we use in-situ holder to heating up the cobalt contained carbon nanofibers to $780{ }^{\circ} \mathrm{C}$. The cobalt-based catalyst with different diameters would generate inside the carbon nanofiber. With the help of high temperature and catalyst nanoparticles, the multilayered graphite crystallites and SWCNTs would grow from large and small NPs, respectively. Meanwhile, by utilizing the ultrahigh time resolution of Gatan K2 in-situ camera, the imperceptible changes could be captured to disclose the growth mechanism of graphite structures.

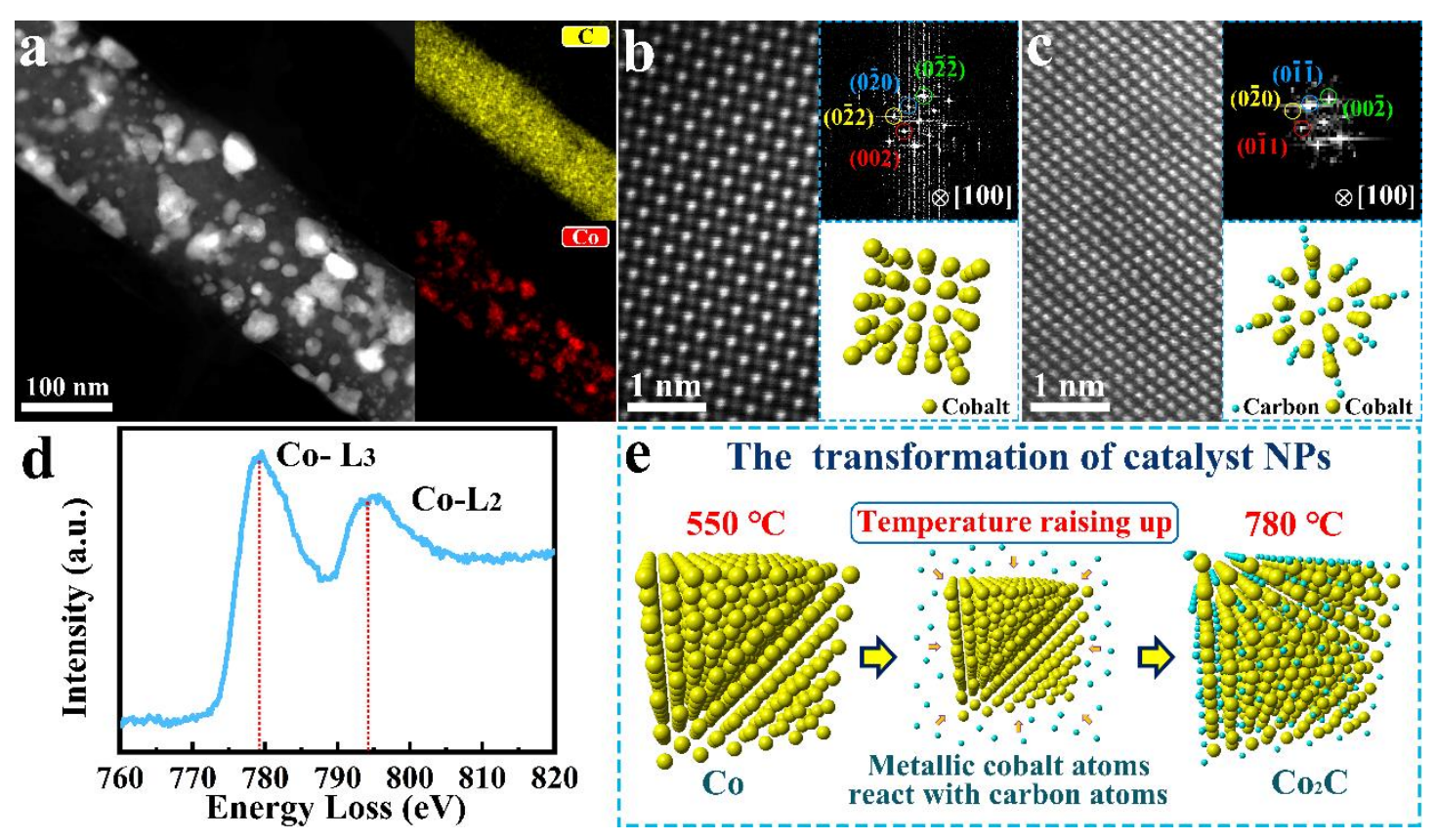


Figure 2. Structural and electronic state characterization of catalyst nanoparticles. (a) Low-magnification STEM image of catalyst NPs contained CNF and EDX elemental mapping of carbon and cobalt. (b)A representative atomic resolution HADDF-STEM image of a single Co NP observed along [100] orientation at $550^{\circ} \mathrm{C}$. (c) A representative atomic resolution HADDF-STEM image of a single $\mathrm{Co}_{2} \mathrm{C}$ NP observed along [100] orientation at $780{ }^{\circ} \mathrm{C}$. (d) A representative EELS spectrum of a cobalt cation column. (e) The schematic diagram of the transformation process of catalyst NPs during the temperature-rise period.

\section{Structural Characterization of Catalyst Nanoparticle}

The catalytic growth of CNTs should be tightly linked to the catalyst structure. In order to fully reveal the structure of catalyst NPs, which actually promote the catalytic graphitization, the temperature was kept at $550{ }^{\circ} \mathrm{C}$ and $780{ }^{\circ} \mathrm{C}$, respectively. These cobalt-based NPs with the diameter from $2 \mathrm{~nm}$ to $40 \mathrm{~nm}$ were generated at $550{ }^{\circ} \mathrm{C}$, which is evidenced by the STEM and EDX elemental mapping images (Figure 2a). As illustrated by the corresponding FFT pattern and unit cell model, the structure of these nascent NPs can be identified a Co fcc structure with [100] orientation (Figure 2b). With the temperature raising up to $780{ }^{\circ} \mathrm{C}$, these metallic NPs were found to transform into $\mathrm{Co}_{2} \mathrm{C}$ NPs, which can be proved by the atomic resolution HADDF-STEM image and the corresponding FFT pattern in Figure 2c. In order to further confirm the correctness of the structural characterization, atomic-column resolution EELS spectra (Figure 1d) collected via line-scan mode from the NP in Figure 2c was used to analyze 
the valence state of the cobalt element. On the basis of the representative EELS spectrum of one Co atomic column $\left(\mathrm{L}_{3} 779.5 \mathrm{eV}\right.$ and $\left.\mathrm{L}_{2} 794.5 \mathrm{eV}\right)$ in Figure 1d (See Figure S3b for EELS spectra of all Co atomic columns on the scanned line, Supporting Information), the first-derivative of this spectrum (Figure S3c) was carried out with the background subtracted and then the $\mathrm{L}_{3}$ and $\mathrm{L}_{2}$ differential peaks were integrated for the ratio calculation. A 4.5 value of $\mathrm{Co}_{3} / \mathrm{L}_{2}$ peak intensity ratio is achieved. Comparing with the reported spectra ${ }^{[32,33]}$, this value unravels a +2 valence state, revealing that the activation of carbon atoms is actually promoted by the valent cobalt ions rather than metallic Co. This transition process indicates that, the NPs inside carbon nanofiber were gradually carbonized under the combined action of dissociative carbon atoms and high temperature during the process of rising to the suitable temperature for catalytic graphitization (Figure 2e). These results should provide an accurate understanding of the catalyst crystal structure and establish a good foundation for further disclosing the mechanism of catalytic graphitization, which will be discussed later in detail. 


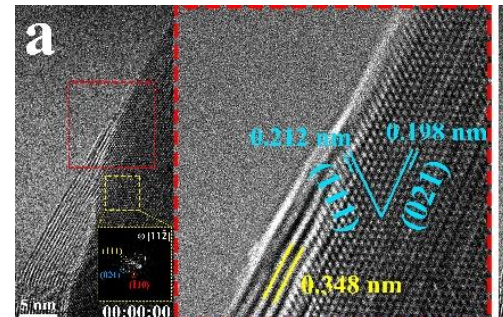

Atomic Steps

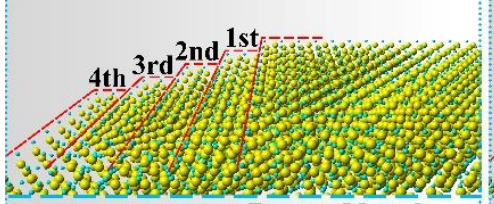

Layer Numbers
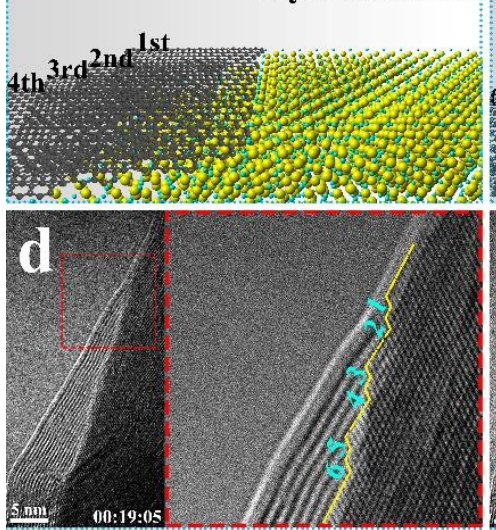

Atomic Steps

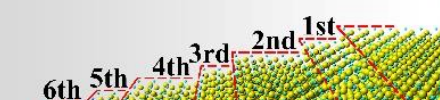

6th 5 th 4 h
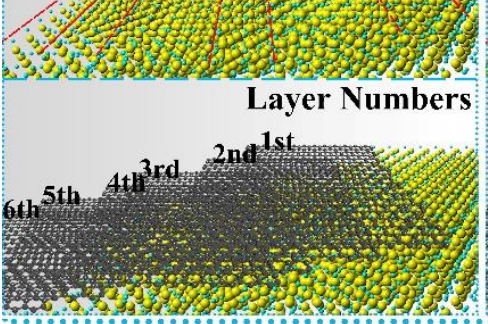

g Step 1

Formation of atomic step

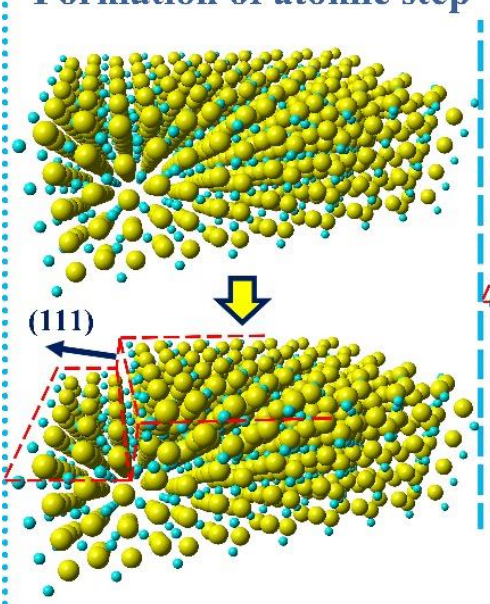

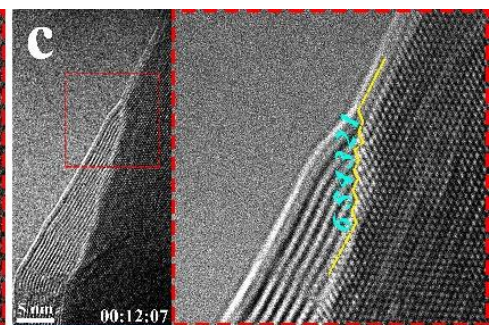

Atomic Steps

Atomic Steps
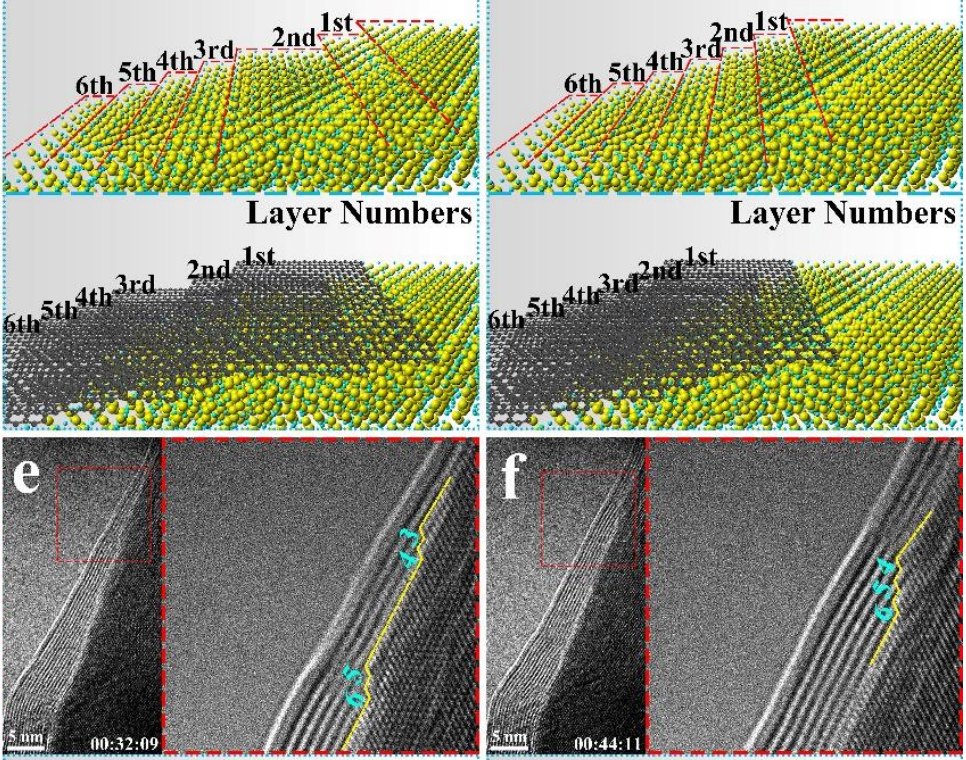

Atomic Steps

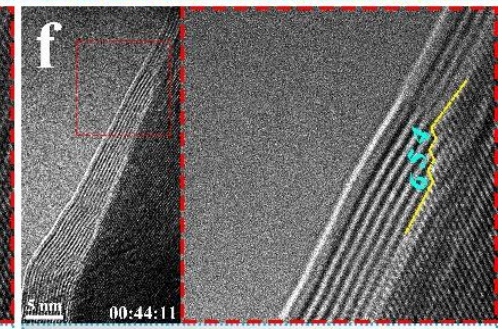

Atomic Steps
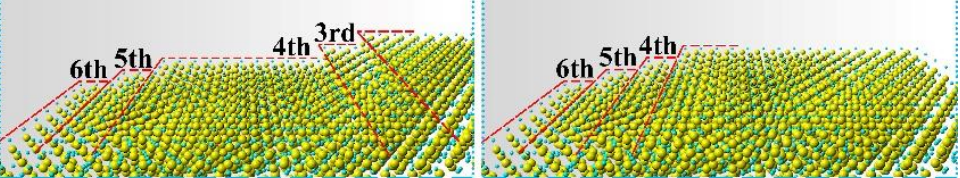

Layer Numbers

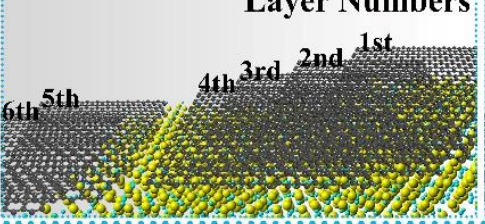

Step 2

Diffusion and Adsorption
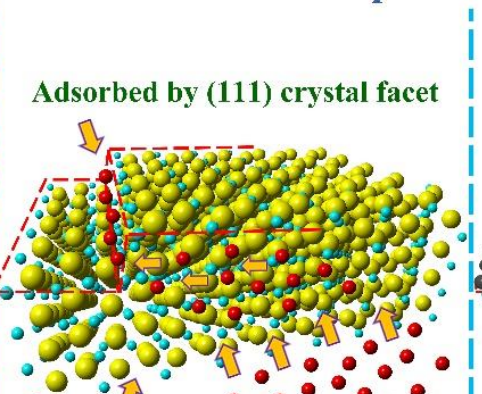

Bulk diffusion

Carbon atoms rich zone
in carbon nanofiber

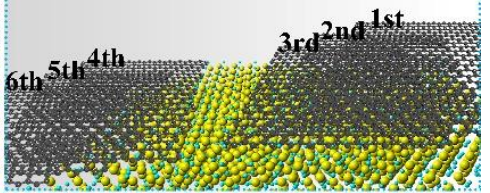

\section{Step 3}

Nucleation and Growth

Nucleation and growth of multilayered graphite crystallite
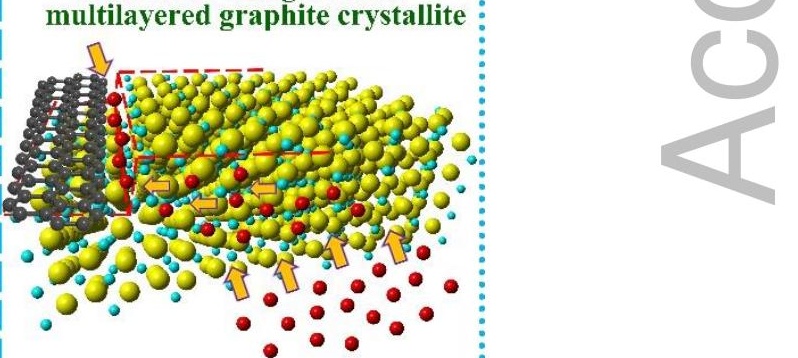

- Dissociative carbon atom

Figure 3. In-situ Cs-TEM observation of the dynamic nucleation and growth process 
of multilayered graphite crystallites from a catalyst $\mathrm{Co}_{2} \mathrm{C}$ NP. (a-f) Sequence of HRTEM images and corresponding schematic diagrams showing the growth stages. (g) The schematic diagrams of "three-steps" growth model.

\section{Nucleation and Growth Process of the Multilayered Graphite Crystallite}

The growth process of the graphite crystallite on the atomic steps of $\mathrm{Co}_{2} \mathrm{C}$ catalyst NP surface can be clearly observed at $780{ }^{\circ} \mathrm{C}$ (as shown in Movie S1). Several representative stages were extracted from the movie and displayed as a series of HRTEM images (Figure 3a, b, c, d, e and f) to anatomize this dynamic process. According to the HRTEM image and corresponding FFT in Figure 3a, we can confirm that the $\mathrm{Co}_{2} \mathrm{C}$ orthorhombic lattice is seen in a [11-2] projection and the atomic steps are generated on the (111) crystal facets. In order to study the nucleation and growth process in detail, the interface between the multilayered graphite crystallites and the atomic steps of the catalyst NP (as indicated by the red dotted frame) is selected. Initially, the layer number of the graphite crystallite were stabilized at four, which was exactly equal to the number of atomic steps, as shown in the schematic diagram at the right bottom of Figure 3a. As the growth progress continues, the graphite crystallites moved forward gradually while the atomic steps retreated with the same rate, and the number of both was consistent (Figure 3b, c and d). Interestingly, we found that the surface width of the second atomic step in Figure $3 b$ increased obviously, which also happened to the second and fifth atomic steps in Figure 3d. Besides, the withdrawal of atomic steps was strictly in the order from the surface to the inside. These phenomena 
demonstrate the asynchronous growth of each layer, which means that the inner layer will continue to grow until the outer layer has already grown for a certain distance. In other words, the graphite crystallites adopt a layer-by-layer growth mode. And due to this growth mode, the first and second layers of graphite crystallites finished the growth process first, and the corresponding atomic steps disappeared completely (Figure 3e). At this point, the surface of the catalyst NP has been wrapped by two layers of graphite crystallites. Subsequently, the third layer of graphite crystallite also completed the growth process in a few seconds (Figure 3f). The successful growth of the third layer means that the $\mathrm{Co}_{2} \mathrm{C}$ catalyst NPs can maintain its catalysis, even if the surface has been encapsulated by the graphite crystallites. This kind of encapsulation process happened on the exposed surface of NP could be more obvious at lower magnification (Figure S4, Supplementary Information). Based on this result, we can basically confirm that for the large NPs with tens of nanometer in diameter, the migration mode of the dissociative carbon atoms is bulk diffusion. Otherwise, the surface diffusion cannot allow the carbon atoms to penetrate the interface of graphite crystallite and catalyst NP, which prevents the formation of multilayered structure. When the carbon atoms migrate to the surface through the catalyst NP, they will be captured by the atomic steps on the (111) crystal facets, and then nucleate and grow into layered graphite crystallites with the help of cobalt ions and high temperature. Accordingly, a "three-steps" growth model (Figure $3 \mathrm{~g})$ could be proposed to clarify the growth mechanism of a multilayered graphite crystallite from the catalyst NP. It is worth noting that, because the lack of atomic steps, the layer number of graphite crystallite at the bottom left corner was kept at fourteen 
throughout the whole growth process. Combined with the growth phenomenon occurring in the atomic step rich region, it can be inferred that the atomic step on the surface of $\mathrm{Co}_{2} \mathrm{C}$ catalyst NP is the essential condition. Meanwhile, the formation of the atomic steps, which act as the active nucleation sites, is attributed to the dynamic selfreshaping of the catalyst NP. In summary, the bulk diffusion of carbon atoms and the dynamic self-reshaping of catalyst NPs jointly promote the layer-by-layer growth of graphite structures catalyzed by large diameter $\mathrm{Co}_{2} \mathrm{C}$ NPs, and directly leads to the formation of multilayered graphite crystallites which closely adhere to the surface of the catalyst NPs. Therefore, the hollow tubular structure cannot be seen to grow outward. 


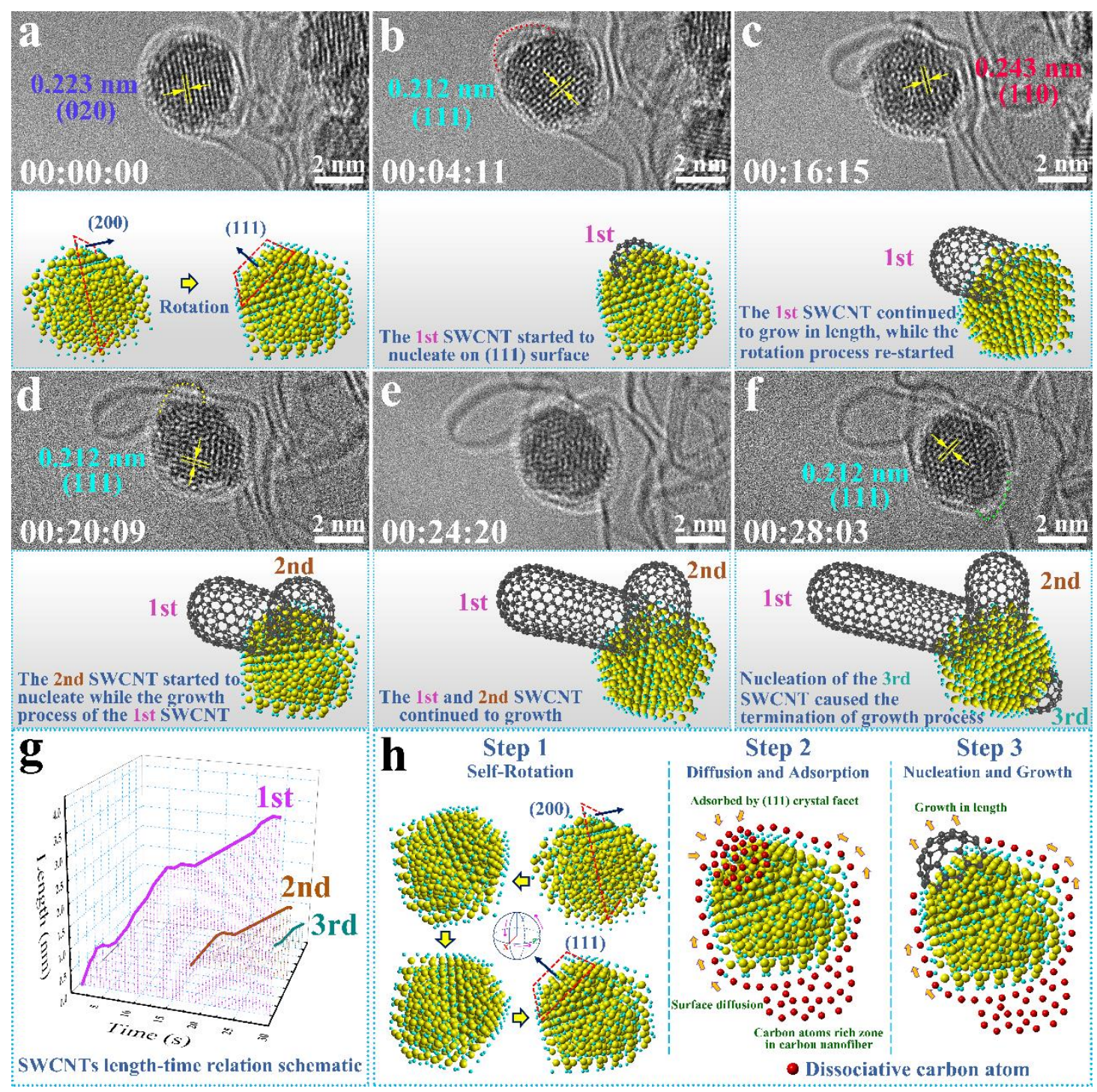

Figure 4. In-situ Cs-TEM observation of the dynamic nucleation and growth process of a globular SWCNT from a catalyst $\mathrm{Co}_{2} \mathrm{C}$ NP. (a-f) Sequence of HRTEM images and corresponding schematic diagrams showing the growth stages. (g) The relation schematic of tube length and time. (h) The schematic diagrams of "three-steps" growth model.

\section{Nucleation and Growth Process of the Single-walled Carbon Nanotube}

The growth of single-walled CNT can be observed at the same temperature when the diameter of catalyst NP reduced to $5.5 \mathrm{~nm}$ (Movie S2, Supporting Information). Before 
the nucleation and growth process, the differences of crystal spacing and orientation in Figure $4 \mathrm{a}$ and $\mathrm{b}$ reveal that the exposure of (111) crystal facet was assisted by a dynamic rotation of the $\mathrm{Co}_{2} \mathrm{C} \mathrm{NP}$, as shown in the schematic diagram at the bottom of Figure 4a. When the restructuring finished, a carbon cap (indicated by red dashed line) gradually nucleated at the upper left part of the $\mathrm{Co}_{2} \mathrm{C}$ NP (Figure 4b) and the axial direction of the carbon cap was perpendicular to the (111) crystal facets. After that, this carbon cap started to grow into a SWCNT with the diameter of $2 \mathrm{~nm}$, which can be proved by the variation in length (Figure 4c). Interestingly, the catalyst NP was found to rotate continuously after finishing the nucleation process, which means that the self-rotation of NP has no effect on the growth of SWCNT. With the length of SWCNT increasing to $3.2 \mathrm{~nm}$, the rotation of catalyst crystal re-exposed the (111) facet and then another carbon cap (indicated by yellow dashed line) nucleated at the top of the NP (Figure 4d). As the first and second SWCNTs continue to grow, the interfaces between these two SWCNTs and NP were found to be on different places (Figure 4e). The imaging principle of TEM creates the illusion that these two SWCNTs are overlapping, however, the top of the second SWCNT was outside the first one and the complete tube walls of them obviously indicate that the nucleation sites of these two tubes should be different. After 4 seconds growth, the crystal rotation made the (111) facet expose again (Figure 4f), since the upper surface of the NP has already been filled with two SWCNTs, which caused the lack of nucleation site, the third carbon cap (indicated by green dashed line) was forced to nucleate at the lower right part. The reason why the first SWCNT was not nucleated at the lower right part of the NP, which had a higher carbon source 
concentration, is because that compared with the vacuum-catalyst interface, the carbon cap has to overcome higher resistance and energy consumption to nucleate and grow at the nanofiber-catalyst interface, which makes the vacuum-catalyst interface to be preferred. And the other interface will be selected until the first kind is filled with grown SWCNTs. The appearance of the third carbon cap directly isolated the NP from the carbon source, rendering the carbon source unable to contact the surface of the catalyst through diffusion and ultimately leads to the termination of the growth process. It is worth noting that the nucleation and growth of the second tube occupied a part of carbon source and further leads to a decrease in growth rate of the first one, meanwhile, since the diffusion rate of the carbon source is substantially unchanged, its own growth rate is also relatively slow, which could be reflected by the relation schematic of tube length and time as shown in Figure 4g. At last, this $\mathrm{Co}_{2} \mathrm{C}$ NP successfully catalyzed the growth of two SWCNTs with $4.5 \mathrm{~nm}$ and $1.8 \mathrm{~nm}$ in length, respectively, and also an uncompleted tube which only existed in the form of carbon cap. In addition, by analyzing the crystal structure of the $\mathrm{Co}_{2} \mathrm{C} \mathrm{NP}$ during the nucleation process, we found that only when the (111) facet are exposed, a new carbon cap will nucleate on it. And other facets, such as (200) and so on, are unable to efficiently catalyze the nucleation process. Combined with structural analysis of catalyst NP during the growth of the multilayered graphite crystallite on the atomic steps of (111) facets, it can be inferred that the (111) crystal facets of $\mathrm{Co}_{2} \mathrm{C}$ NPs have the highest catalytic activity in catalyzing the nucleation of the carbon cap. Meanwhile, five critical information can be obtained from the whole dynamic growth process: (1) There is no significant change in the size 
and shape of the catalyst NP; (2) The surface of the NP is smooth, in other words there is no obvious atomic step at the nucleation sites of carbon caps; (3) The growth rate of SWCNT cannot be influenced by the self-rotation of catalyst NP, which indicates that the diffusion rate and direction of carbon atoms does not change with the crystal rotation; (4) The position where the SWCNT has been grown will no longer have a new carbon cap nucleation; (5) When the third carbon cap isolated the carbon source from the catalyst, the growth process would stop rapidly. According to the above results, we strongly suggest that for those $\mathrm{Co}_{2} \mathrm{C}$ catalyst NPs with the diameter of several nanometers, the surface diffusion of carbon atoms is dominant, which is quite different from the bulk diffusion in those large NPs with tens of nanometers in diameter. Otherwise, the bulk diffusion of carbon atoms will cause the deformation of catalyst NP and also the re-nucleation of new carbon caps inside the already grown tubes, which would further form the multi-walled carbon nanotubes. According to these observations and analyses, a "three-steps" growth model (Figure 4h) could be proposed to clarify the growth mechanism of SWCNTs from the catalyst NP with several nanometers in diameter. At the first, before the nucleation process, the structural rotation of $\mathrm{Co}_{2} \mathrm{C} \mathrm{NP}$ causes the exposure of the (111) crystal facet. Then those carbon atoms diffuse through the surface of catalyst NP are captured by the (111) facet to precipitate. After the diffusion and adsorption step, a newborn carbon cap will nucleate with the help of cobalt ions and high temperature, and the continuous supply of the carbon atoms will help the carbon cap grow into a SWCNT. 


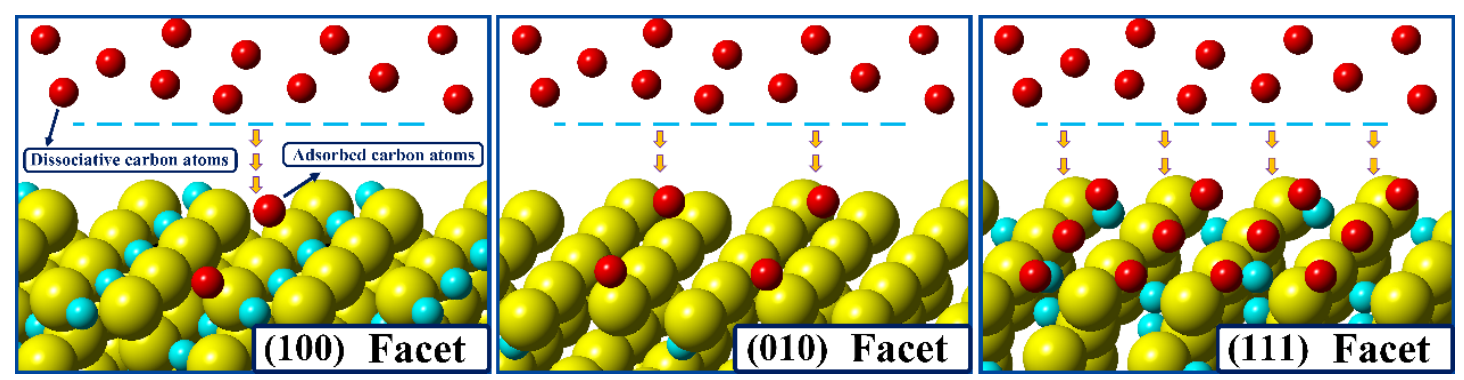

Figure 5. The schematic diagrams of dissociative carbon atoms adsorbed on (100), (010) and (111) crystal facets of $\mathrm{Co}_{2} \mathrm{C}$ catalyst NP.

Table 1. DFT calculations to determine the adsorption energy (eV) of carbon atoms on various crystal facets of $\mathrm{Co}_{2} \mathrm{C}$

\begin{tabular}{|c|c|c|c|}
\hline Crystal Facets $^{(\mathrm{a})}$ & $(100)$ & $(010)$ & (111) \\
\hline Adsorption Energy $(\mathrm{eV})^{(\mathrm{b})}$ & 6.53 & 5.74 & 5.12 \\
\hline
\end{tabular}

(a-b) The DFT calculations of adsorption energy based on the configurations of dissociative carbon atoms adsorbed on three crystal facets of $\mathrm{Co} 2 \mathrm{C}$ catalyst NP.

\section{Calculation of the Adsorption Energy}

In order to comprehensively analyze the relationship between the selective nucleation and crystal facet, we tracked the growth processes on more than 50 NPs with different diameter. We found that whether the multilayered graphite crystallites or the carbon caps preferentially grow on the $\mathrm{Co}_{2} \mathrm{C}$ (111) facet rather than other crystal facets. The density function theory (DFT) was employed to further understand the mechanism of spontaneous crystal facet selection. The calculations of adsorption energies are simplified through the replacement of complete graphite structure by carbon atoms. The crystal facets of (110), (101) and (011) are ignored in our calculation because their 
surface energies $\left(161,129\right.$ and $135 \mathrm{meV} / \AA^{2}$, respectively) are lower than those of $(100)$, (001) and (111) facets (162, 170 and $170 \mathrm{meV} / \AA^{2}$, respectively), which have less probability for the nucleation of graphite structures ${ }^{[34]}$. Besides, (010) facet is also ignored because the (010) and (001) facets are exactly same according to the crystal symmetry. Figure 5 a shows the schematic diagrams of dissociative carbon atoms adsorbed on (100), (010) and (111) crystal facets of $\mathrm{Co}_{2} \mathrm{C}$ catalyst NP. The adsorption energies of a single carbon atom are calculated to be $6.53,5.74$ and $5.12 \mathrm{eV}$ for (100), (010) and (111) facets, respectively (Table 1). Obviously, it could be easier for (111) facets to capture the dissociative carbon atoms and further promote the nucleation of graphite structures. Therefore, the (111) facets were observed to be the preferred crystal facet in above experiment.

\section{Conclusions}

The nucleation and growth process of graphite structures catalyzed by $\mathrm{Co}_{2} \mathrm{C}$ NPs were directly observed by taking advantage of in-situ Cs-TEM technique at low accelerating voltage of $80 \mathrm{kV}$, which eliminates the effects of electron beam irradiation to the greatest extent. Our observations reveal that catalyst NP with $5.5 \mathrm{~nm}$ diameter satisfies the conditions for growing SWCNTs and the catalytic reaction is actually promoted by the valent cobalt ions rather than metallic Co. Besides, the growth of multilayered graphite crystallites on the large catalyst NP with tens of nanometers suggests that the size of NP influences the diffusion mode of carbon atoms, which directly determines the type of catalytic products. Whether the carbon atoms adopt 
surface or bulk diffusion, the nucleation of graphite structures preferentially selects the (111) crystal facets of $\mathrm{Co}_{2} \mathrm{C}$ NPs because of lower adsorption energy which is proved by the DFT calculations. Our findings should provide a deep insight into the dynamic process of graphite structural growth and be significant for realizing efficient and controllable synthesis of high-quality CNTs.

\section{Experimental Section}

Sample Synthesis. Cobalt contained carbon nanofibers were prepared by using electrospinning method. The electrospinning electrolyte was mixed by $6 \mathrm{ml}$ solution A and $4 \mathrm{ml}$ solution $\mathrm{B}$. The solution A was composed of $1.0 \mathrm{~g}$ polyacrylonitrile (PAN)

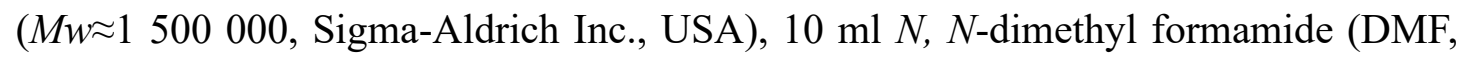
A.R., Tianjin Chemical Corp., China). The solution B was composed of $0.8 \mathrm{~g}$ cobalt nitrate $\left(\mathrm{Co}\left(\mathrm{NO}_{3}\right)_{2} \cdot 6 \mathrm{H}_{2} \mathrm{O}\right.$, A.R., Alfa-Aesar Inc., USA), 0.375 g polyvinylpyrrolidone (PVP) (Mw $\approx 1300$ 000, Sigma-Aldrich Inc., USA) and $4 \mathrm{ml} N, N$-dimethyl formamide (DMF, A.R., Tianjin Chemical Corp., China). The electrospinning process was performed at $12.5 \mathrm{kV}$ DC voltage, $12.5 \mathrm{~cm}$ electrode gap between needle tip and collector, and a feed rate of $0.5 \mathrm{~mL} \cdot \mathrm{h}^{-1}$ in a dedicated electrospinning setup. The preoxidation was carried out at $240{ }^{\circ} \mathrm{C}$ for $4 \mathrm{~h}$ in the air with a $2{ }^{\circ} \mathrm{C} / \mathrm{min}$ heating rate and then naturally cooled down to the room temperature.

Characterization Methods. The crystal structure of $\mathrm{Co}_{2} \mathrm{C}$ was characterized by a probe aberration-corrected scanning transmission electron microscope (Titan Themis G2 300, FEI, USA) either in a high-resolution mode (HRTEM) or with a high angle 
annular dark field detector in a scanning TEM mode (STEM-HAADF). The valance states of cobalt atoms were identified at the atomic scale by electron energy loss spectrum (EELS) line scan. The in-situ experiments were performed under $80 \mathrm{kV}$ in HRTEM mode by using a commercial heating holder (FEI NanoEx-i/v-TEM holder, FEI, USA). Both growth evolution of CNTs and phase transition of the catalyst $\mathrm{Co}_{2} \mathrm{C}$ NPs were recorded by high speed K2 IS Camera (Gatan K2 In-Situ camera, Gatan, USA).

Computational Method. The adsorption energies of $\mathrm{C}_{6}$ on the $\mathrm{Co}_{2} \mathrm{C}$ crystal facets were calculated by the Vienna Ab-initio Simulation Package ${ }^{[35,36]}$ (VASP), taking advantage of the density functional theory (DFT) and the Projected Augmented Wave ${ }^{[37]}$ (PAW) method. The Perdew-Burke-Ernzerhof (PBE) functional was used to describe the exchange and correlation effect. ${ }^{[38]}$ For all the geometry optimizations, the cutoff energy was set to be $450 \mathrm{eV}$. Spin-polarization was included in all the cases. The MonkhorstPack grids ${ }^{[39]}$ were set to be $3 \times 3 \times 1,3 \times 2 \times 1$, and $3 \times 3 \times 1$ for performing the calculations on the (100), (010), and (111) facets of $\mathrm{Co}_{2} \mathrm{C}$, respectively. The adsorption energy was defined as

$$
\Delta \mathrm{E}_{a d s}=E_{a d s}-E_{\text {slab }}-6 E_{C}
$$

where $E_{a d s}$ is the electronic energy of the facet with an adsorbed $\mathrm{C}_{6}$ species, $E_{s l a b}$ is the electronic energy of the clean surface, and $E_{C}$ is the electronic energy of one carbon atom referenced to graphene. Under this definition, a smaller value indicates a stronger adsorption system. 


\section{Acknowledgements}

This work was supported by National Natural Science Foundation of China (51571104, 51771085, 11604130, 51601082 and 11274145), MOST International Cooperation Funds (2014DFA91340), the Fundamental Research Funds for the Central Universities (lzujbky-2017-176, lzujbky-2017-25 and lzujbky-2017-177) and Open Project of Key Laboratory of Magnetism and Magnetic Materials of the Ministry of Education, Lanzhou University (LZUMMM2017003).

\section{Conflict of Interest}

There are no conflicts of interest to declare.

\section{References}

[1] A. D. Franklin, Z. Chen, Nat. Nanotechnol. 2010, 5, 858-862.

[2] D.-M. Sun, M. Y. Timmermans, Y. Tian, A. G. Nasibulin, E. I. Kauppinen, S. Kishimoto, T. Mizutani, Y. Ohno, Nat. Nanotechnol. 2011, 6, 156-161.

[3] T. Yamada, Y. Hayamizu, Y. Yamamoto, Y. Yomogida, A. Izadi-Najafabadi, D. N. Futaba, K. Hata, Nat. Nanotechnol. 2011, 6, 296-301.

[4] D. Zhong, Z. Zhang, L. Ding, J. Han, M. Xiao, J. Si, L. Xu, C. Qiu, L.-M. Peng, Nat. Electron. 2018, 1, 40-45.

[5] A. Ishii, X. He, N. F. Hartmann, H. Machiya, H. Htoon, S. K. Doorn, Y. K. Kato, Nano Lett. 2018, 18, 3873-3878.

[6] X. He, H. Htoon, S. K. Doorn, W. H. P. Pernice, F. Pyatkov, R. Krupke, A. Jeantet, 
Y. Chassagneux, C. Voisin, Nat. Mater. 2018, 17, 663-670.

[7] Q. Wen, R. Zhang, W. Qian, Y. Wang, P. Tan, J. Nie, F. Wei, Chem. Mater. 2010, 22, 1294-1296.

[8] C. L. Cheung, A. Kurtz, H. Park, C. M. Lieber, J. Phys. Chem. B 2002, 106, 24292433.

[9] K. Hata, D. N. Futaba, K. Mizuno, T. Namai, M. Yumura, S. Iijima, Science 2004, $306,1362-1364$.

[10] A. R. Harutyunyan, G. Chen, T. M. Paronyan, E. M. Pigos, O. A. Kuznetsov, K. Hewaparakrama, S. M. Kim, D. Zakharov, E. A. Stach, G. U. Sumanasekera, Science 2009, 326, 116-120.

[11] W. Kim, H. C. Choi, M. Shim, Y. Li, D. Wang, H. Dai, Nano Lett. 2002, 2, 703708.

[12] W.-H. Chiang, R. M. Sankaran, Nat. Mater. 2009, 8, 882-886.

[13] X. Li, X. Tu, S. Zaric, K. Welsher, W. S. Seo, W. Zhao, H. Dai, J. Am. Chem. Soc. 2007, 129, 15770-15771.

[14] W.-H. Chiang, M. Sakr, X. P. A. Gao, R. M. Sankaran, ACS Nano 2009, 3, 40234032.

[15] M. He, A. I. Chernov, P. V. Fedotov, E. D. Obraztsova, J. Sainio, E. Rikkinen, H. Jiang, Z. Zhu, Y. Tian, E. I. Kauppinen, M. Niemelä, A. O. I. Krause, J. Am. Chem. Soc. 2010, 132, 13994-13996.

[16] H. Wang, B. Wang, X.-Y. Quek, L. Wei, J. Zhao, L.-J. Li, M. B. Chan-Park, Y. Yang, Y. Chen, J. Am. Chem. Soc. 2010, 132, 16747-16749. 
[17] F. Yang, X. Wang, D. Zhang, J. Yang, D. Luo, Z. Xu, J. Wei, J.-Q. Wang, Z. Xu, F. Peng, X. Li, R. Li, Y. Li, M. Li, X. Bai, F. Ding, Y. Li, Nature 2014, 510, 522-524.

[18] S. Zhang, L. Kang, X. Wang, L. Tong, L. Yang, Z. Wang, K. Qi, S. Deng, Q. Li, X. Bai, F. Ding, J. Zhang, Nature 2017, 543, 234-238.

[19] X. Feng, S. W. Chee, R. Sharma, K. Liu, X. Xie, Q. Li, S. Fan, K. Jiang, Nano Res. 2011, 4, 767.

[20] L. Zhang, M. He, T. W. Hansen, J. Kling, H. Jiang, E. I. Kauppinen, A. Loiseau, J. B. Wagner, ACS Nano 2017, 11, 4483-4493.

[21] S. Helveg, C. López-Cartes, J. Sehested, P. L. Hansen, B. S. Clausen, J. R. RostrupNielsen, F. Abild-Pedersen, J. K. Nørskov, Nature 2004, 427, 426-429.

[22] M. Lin, J. P. Y. Tan, C. Boothroyd, K. P. Loh, E. S. Tok, Y.-L. Foo, Nano Lett. 2006, $6,449-452$.

[23] S. Hofmann, R. Sharma, C. Ducati, G. Du, C. Mattevi, C. Cepek, M. Cantoro, S. Pisana, A. Parvez, F. Cervantes-Sodi, A. C. Ferrari, R. Dunin-Borkowski, S. Lizzit, L. Petaccia, A. Goldoni, J. Robertson, Nano Lett. 2007, 7, 602-608.

[24] H. Yoshida, S. Takeda, T. Uchiyama, H. Kohno, Y. Homma, Nano Lett. 2008, 8, 2082-2086.

[25] S. Hofmann, R. Blume, C. T. Wirth, M. Cantoro, R. Sharma, C. Ducati, M. Havecker, S. Zafeiratos, P. Schnoerch, A. Oestereich, D. Teschner, M. Albrecht, A. Knop-Gericke, R. Schlögl, J. Robertson, J. Phys. Chem. C 2009, 113, 1648-1656. [26] C. T. Wirth, B. C. Bayer, A. D. Gamalski, S. Esconjauregui, R. S. Weatherup, C. Ducati, C. Baehtz, J. Robertson, S. Hofmann, Chem. Mater. 2012, 24, 4633-4640. 
[27] D.-M. Tang, C. Liu, W.-J. Yu, L.-L. Zang, P.-X. Hou, J.-C. Li, F. Li, Y. Bando, D. Golberg, H.-M. Cheng, ACS Nano 2014, 8, 292-301.

[28] F. B. Romdhane, J. A. Rodríguez-Manzo, A. Andrieux-Ledier, F. Fossard, A. Hallal,

L. Magaud, J. Coraux, A. Loiseau, F. Banhart, Nanoscale 2016, 8, 2561-2567.

[29] T. Ichihashi, J.-i. Fujita, M. Ishida, Y. Ochiai, Phys. Rev. Lett. 2004, 92, 215702.

[30] J. A. Rodríguez-Manzo, M. Terrones, H. Terrones, H. W. Kroto, L. Sun, F. Banhart, Nat. Nanotechnol. 2007, 2, 307-311.

[31] A. Kis, G. Csányi, J.-P. Salvetat, T.-N. Lee, E. Couteau, A. J. Kulik, W. Benoit, J. Brugger, L. Forró, Nat. Mater. 2004, 3, 153-157.

[32] Z.-L. Wang, J.-S. Yin, Y.-D. Jiang, Micron 2000, 31, 571-580.

[33] P. Müller, M. Meffert, H. Störmer, D. Gerthsen, Microsc. Microanal. 2013, 19, $1595-1605$.

[34] Y.-H. Zhao, H.-Y. Su, K. Sun, J. Liu, W.-X. Li, Surf. Sci. 2012, 606, 598-604.

[35] G. Kresse, J. Furthmüller, Phys. Rev. B 1996, 54, 11169.

[36] G. Kresse, J. Hafner, Phys. Rev. B 1994, 49, 14251.

[37] P. E. Blöchl, Phys. Rev. B 1994, 50, 17953.

[38] J. P. Perdew, K. Burke, M. Ernzerhof, Phys. Rev. Lett. 1996, 77, 3865.

[39] H. J. Monkhorst, J. D. Pack, Phys. Rev. B 1976, 13, 5188. 


\section{Table of Contents}

The catalytical growth mechanism of graphite structures from $\mathrm{Co}_{2} \mathrm{C}$ nanoparticles was directly investigated by in-situ Cs-TEM under low accelerating voltage of $80 \mathrm{kV}$ at $780{ }^{\circ} \mathrm{C}$. The multilayered graphite crystallites prefer to grow on the nanoparticles with tens nanometers in diameter. When the diameter of nanoparticles reduces to several nanometers, the growth of single-walled carbon nanotubes could be observed.

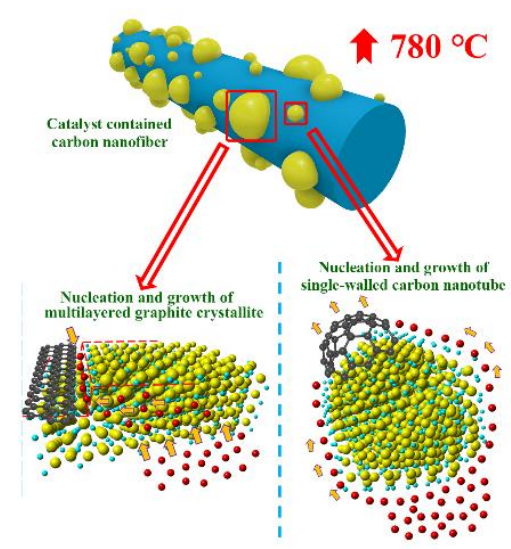

Keywords: in-situ electron microscope. carbide nanoparticle. graphite structure. nucleation and growth mechanism. adsorption energy 